\title{
The aqueous extract of cinnamon bark ameliorated cisplatin-induced cytotoxicity in vero cells without compromising the anticancer efficiency of cisplatin
}

\author{
Ayman I. ElKady ${ }^{a, b}$, Wafaa S. Ramadan ${ }^{c, d}$
}

\begin{abstract}
Aim. Cis-diammine dichloroplatinum (CDDP) is one of the most important chemotherapeutic agents for cancer treatment. Nonetheless, its notable side effect, nephrotoxicity, undermines its clinical use. The current study was undertaken to evaluate the protective potential of the aqueous extract (AEC) of Cinnamomum cassia (cinnamon) against the cytotoxic effect of CDDP in vitro and to elaborate the molecular mechanism underlying protection.

Methods. MTT assay was performed to assess viability of the normal kidney Vero cells treated with CDDP and/or AEC. Cells were stained with Coomassie blue, acridine orange and ethidium bromide to highlight morphological features of apoptosis. Caspase-3 activity, DNA fragmentation and reactive oxygen species (ROS) level were monitored to assess biochemical hallmarks of apoptosis. Quantitative RT-PCR and Western blot analyses were performed to elucidate expression of cellular molecules underlying the protective potential of AEC.

Results. CDDP-treated Vero cells exhibited hallmarks of apoptosis; these hallmarks were significantly suppressed in the presence of AEC. AEC did not alter activity of CDDP-induced cytotoxicity of breast and liver cancer cells. AEC treatment of Vero cells prevented CDDP-induced increased expression of mitochondrial Bax protein, release of mitochondrial cytochrome c, caspase-3 activation, DNA fragmentation and generation of ROS. AEC up-regulated expression of the cytoprotective gene (heme oxygenase (HO)-1).

Conclusion. These findings suggest AEC has protective effects against CDDP-induced toxicity via preventing the activation of various cellular mechanisms mediating apoptotic cell death, without compromising the anticancer efficiency of CDDP. Thus, cinnamon may represent one of the most feasible ways to reduce the risk of CDDP-induced toxicity.
\end{abstract}

Key words: apoptosis, cinnamon, cisplatin, nephrotoxicity, vero cells, cytoprotective genes

Received: April 22, 2016; Accepted: June 17, 2016; Available online: July 25, 2016

http://dx.doi.org/10.5507/bp.2016.034

${ }^{a}$ Department of Biological Sciences, Faculty of Sciences, King Abdulaziz University, Jeddah, Saudi Arabia

${ }^{b} Z$ oology Department, Faculty of Science, Alexandria University, Alexandria, Egypt

'Department of Anatomy, Faculty of Medicine, King Abdulaziz University, Jeddah, Saudi Arabia

${ }^{d}$ Department of Anatomy, Faculty of Medicine, Ain Shams University, Cairo, Egypt

Corresponding author: Ayman I. ElKady, e-mail:aielkady@yahoo.co.uk

\section{INTRODUCTION}

Cis-diamminedichloroplatinum, Cisplatin (CDDP), is one of the most widely used chemotherapeutic agents for the treatment of several human malignancies. Nonetheless, its efficacy is often limited by its side effects as it may induce serious toxicity in normal tissues and organs including the kidneys ${ }^{1}$. Various approaches have been attempted to curtail these side effects such as using the novel CDDP analogues and hydrating the patients during CDDP treatment ${ }^{2}$. Despite these efforts, CDDP-induced nephrotoxicity remains a major obstacle that limits its use and efficacy in cancer therapy ${ }^{3}$. The cytotoxic effects of CDDP are postulated to occur via several mechanisms, including inhibition of protein synthesis, mitochondrial injury, and DNA damage, which lead ultimately to activation of apoptotic pathways in tumor cells as well as renal tubule cells ${ }^{4}$. Two major apoptotic pathways have been implicated in CDDP-nephrotoxicity: the mitochondrial (intrinsic) and the cell death receptor (extrinsic) pathways. Both extrinsic and intrinsic path- ways eventually converge on a common execution phase of apoptosis (activation of caspase-3) that provokes engagement of the effector caspases ${ }^{5}$. On the activation of the latter caspase, the cleavage of cellular machineries essential for cell viability cascades. This results in shrinkage of the cell and fragmentation into membrane-bound apoptotic bodies, which are eventually subjected to rapid phagocytosis by the surrounding cells ${ }^{6}$. Therefore, new approaches, such as novel effective drugs with mild toxicities or excellent combination regimens, are needed to ameliorate CDDP side effects.

Cumulative studies demonstrate there are substances commonly used by cancer patients to either "treat" cancer and/or "reduce the toxicity" induced by chemotherapy? Indeed, a growing list of studies document that patients receiving medicinal herbs with chemotherapy or radiation do not only experience fewer side effects, but also have better short-term and long-term outcomes in relation to their disease ${ }^{8}$. For example, the extracts of Chinese medicinal herbs are often used together with traditional cancer therapy to improve the survival rate and quality 
of life, as the use of herbal extracts are much less expensive than the standard antineoplastic therapies currently available ${ }^{9,10}$. Cinnamon (Cinnamomum cassia), belonging to the Lauracease family, is a favorite spice around the world, owing to its flavor, health benefits and wide uses in traditional medicine ${ }^{11}$. The chemical constituent of cinnamon bark includes $65-80 \%$ Cinnamaldehyde and $5-10 \%$ Eugenol, in addition to other compounds present in lesser percentages such as cinnamic acid, hydroxyl cinnamaldehyde, cinnamyl alcohol, coumarin and cinnamyl acetate ${ }^{12}$. Volatile oils from the different parts of cinnamon including leaves, bark, fruits, root flowers, and buds have been isolated. Despite the isolation of several components in cinnamon and the appreciation of their therapeutic application have been demonstrated, it was found that using the whole plant helps to decrease the side effects that may occur on using the isolated components ${ }^{13}$. The aqueous and alcoholic extracts of cinnamon have been found to potentially inhibit fatty acid oxidation and lipid peroxidation in vitro ${ }^{14}$. Different flavonoids isolated from cinnamon have free-radical-scavenging activities and antioxidant properties ${ }^{15}$. It was revealed that cinnamaldehyde possesses potential activity against the production of nitric oxide as well as the expression of inducible nitric oxide. Furthermore, in a comparative study cinnamon phenolic extract showed the highest antioxidant activity, among screened 26 herbal extracts ${ }^{16}$. In addition, cinnamon possesses anti-inflammatory, anti-cancer, antiangiogenesis antimicrobial, and antidiabetic activities ${ }^{17-19}$.

Based on the above studies valuing cinnamon properties, this herb provides a fertile ground for future investigations and discoveries pertinent to its use as an ameliorating agent against CDDP cellular toxicity. Thus far, studies on the combined effect of cinnamon and CDDP are lacking and not convincing enough to allow a general recommendation for using both agents in a combination regimen. This study provides a platform for evaluating the potentiality of cinnamon to counter the effect of CDDP-induced toxicity of kidney cells. We hypothesized that since cinnamon extract involves a wide array of ingredients that have substantially different biochemical characteristics, these ingredients may simultaneously target multiple molecular and cellular pathways involved in the process of CDDP-induced cyto-toxicity.

\section{MATERIALS AND METHODS}

\section{Preparation of aqueous extract of Cinnamon}

Cinnamon bark (100 mg), purchased from local markets, was cleaned, ground and mixed with $1000 \mathrm{ml}$ of distilled water and the mixture was boiled at $100{ }^{\circ} \mathrm{C}$ for $30 \mathrm{~min}$. The decoction was centrifuged, filtered using 0.2 mm Millipore filter (St. Quentin en Yvelines, France), lyophilized (Lypholizer model: Christ -alpha 1-2LD plusGermany), re-dissolved in DMSO to the desired concentrations and denoted AEC ( ref. $^{20}$ ).

\section{Gas chromatography-mass spectrometry (GC-MS)}

Analysis of the decoction of cinnamon was performed using a GCMS-QP2010 plus (Shimadzu, Kyoto, Japan) and a DB-5 column $(30 \mathrm{~m} \times 0.25 \mathrm{~mm} \times 0.25 \mu \mathrm{m})(\mathrm{J} \& \mathrm{~W}$ Scientific, Folsom, CA) was included in the gas chromatograph system ${ }^{21}$.

\section{High-performance liquid chromatography (HPLC)}

The combined diethyl ether layers were evaporated and the resultant residue was dissolved in HPLC grade Methanol (MeOH) to give 1.000 ppm. HPLC YL 9100 system (Younglin, Anyang, Korea) was used. Identification of the phenolic compounds in the sample was determined by comparing the retention times of known peaks. Column C/8, Ultraviolet (UV) $220 \mathrm{~nm}$, Refractive Index $(\mathrm{RF})=1\left(\right.$ ref. $\left.^{22}\right)$

\section{Culture of Vero cells}

The Vero cell line, an immortal, non-oncogenic fibroblastic cell line established from kidney cells of the African green monkey (Cercopithecus aethiops) has been used to study cell growth, differentiation ${ }^{23}$ and cytotoxicity $^{24}$. Vero cells were cultured in Dulbecco's modified eagle's medium (DMEM) (Biochrom, Germany) supplemented with $10 \%$ FCS, $100 \mu \mathrm{g} / \mathrm{mL}$ Penicillin/streptomycin. Cells were maintained in $5 \% \mathrm{CO} 2-95 \%$ air at $37{ }^{\circ} \mathrm{C}$ and sub-cultured every other day.

\section{Cell growth analysis}

Cell viability and the effects of CDDP (Sigma), and AEC on the growth of Vero cells were assessed by Watersoluble tetrazolium salt-1(WST-1) Cell Proliferation Assay Kit (Cayman chemicals-USA) according to the manufacturer's instructions. Briefly, Vero cells were seeded onto 96-well plates $\left(10^{4}\right.$ cells/well $)$ and grown overnight. The cells were then treated with indicated concentrations of the CDDP and AEC and incubated for $24 \mathrm{~h}$. At the end of each incubation, $10 \mu \mathrm{L}$ of freshly prepared WST-1 solution was added to each well. Culture medium and WST-1/ solution were added in an empty well as a blank for the microtiter plate reader. The absorbance of the treated and untreated samples was measured after $2 \mathrm{~h}$ by a micro plate reader (BioTek Synergy HT), at $450 \mathrm{~nm}$ with a reference wavelength $630 \mathrm{~nm}$ to avoid the interference of cell layer absorbance that blocks light passing through.

\section{Nuclear and cytoplasmic staining assays}

The nuclear morphological changes associated with apoptosis were analyzed using Coomassie stain and fluorescent stains, Hoechst 33342, Acridine orange (Ao) and Ethidium bromide (EtBr). Vero cells were cultured in slide chambers and allowed to adhere to the bottom of the wells for $24 \mathrm{~h}$ before treatment. Cells were then exposed to indicated concentrations of the CDDP and AEC for $24 \mathrm{~h}$, before being washed with PBS and stained with Hoechst 33342 or Ao/EtBr (Sigma) for $15 \mathrm{~min}$ at $37^{\circ} \mathrm{C}$. Subsequently, cells were washed with PBS and viewed under a fluorescence microscope (Axioplan 2 Zeiss, Germany). For Coomassie staining, cells were fixed using 
cold ethanol, washed with PBS, stained with Coomassie examined under an inverted microscope (Model:1X2ILL100-Japan) and then photographed.

\section{Activation of Caspase-3}

Caspase-3/7 activities were determined by using the Apo-ONE® Homogeneous Caspase-3/7 Assay (Promega). Vero cells were seeded on a 96-well luminometer plate $\left(10^{4}\right.$ cells/well $)$ and treated with indicated concentrations of CDDP, AEC, or both for $24 \mathrm{~h}$. Then the activities of Caspase-3/7 were measured according to the manufacturer's instructions. Briefly, $100 \mu \mathrm{L}$ of ApoONE® Caspase-3/7 Reagent was added to each well and the contents of the wells were mixed using a plate shaker at 300-500 rpm for $12 \mathrm{~h}$. The fluorescence of each well was measured using a micro-plate reader (BioTek Synergy) with excitation at 485/20 nm and emission 528/20 nm.

\section{Quantitation of DNA fragmentation}

DNA fragmentation was performed as previously detailed $^{25}$. Briefly, control and treated cells were suspended in $250 \mathrm{uL} 10 \mathrm{~mm}$ Tris and $1 \mathrm{~mm}$ EDTA, pH 8.0 (TE buffer), and were incubated with an additional volume lysis buffer ( $5 \mathrm{~mm}$ Tris, $20 \mathrm{~mm}$ EDTA, pH 8.0, 0.5\% Triton $\mathrm{X}-100$ ) for $30 \mathrm{~min}$ at $4{ }^{\circ} \mathrm{C}$. The intact pellet was separated from DNA fragments (supernatant) by centrifugation for $15 \mathrm{~min}$ at 13,000 g. TE buffer (500 muL) was added to the pellets and precipitation was done by adding $500 \mathrm{muL}$ $10 \%$ trichloroacetic acid at $4{ }^{\circ} \mathrm{C}$. Samples were pelleted at 4000 r.p.m. for $10 \mathrm{~min}$, and the supernatant was removed. Trichloroacetic acid ( $300 \mu \mathrm{L}, 5 \%)$ was added and samples were boiled for $15 \mathrm{~min}$. DNA contents were quantitated using the diphenylamine reagent. Optical density was read at $600 \mathrm{~nm}$ with a multi-well spectrophotometer reader. The percentage of DNA fragmented was calculated as the ratio of the DNA content in the supernatant to the amount in the pellet.

\section{Determination of ROS production}

The intracellular ROS level was measured using a fluorescent dye 2',7' dichlorodihydrofluorescein diacetate (H2-DCFDA; Invitrogen). Cells were plated in 96-well plates and treated as detailed above. After washing with PBS, serum-free DMEM containing $10 \mu \mathrm{M}$ H2-DCFDA was added to each well and incubated at $37^{\circ} \mathrm{C}$ for $1 \mathrm{~h}$. ROS production was measured using a microplate reader equipped with a spectrofluorometer at an emission wavelength of $538 \mathrm{~nm}$ and extinction wavelength of $485 \mathrm{~nm}$.

\section{RNA extraction and quantitative RT-PCR}

For the determination of HO-1 expression level, cells were seeded $\left(20 \times 10^{4} /\right.$ well) onto 6 -well plates and treated with indicated concentrations of CDDP and AEC for 24 h. After this period, floating and adherent cells were collected, washed with PBS and pelleted by centrifugation (700 g, $5 \mathrm{~min}$ ). For RNA extraction and reverse transcriptase-PCR, gene-specific primers were used as previously described $^{26,27}$.

\section{Western blot analysis}

For the determination of Bax and cytochrome c expression levels by Western blot analyses, polyacrylamide gel electrophoresis and immunoblots were performed as previously described ${ }^{26}$.

\section{Statistical analysis}

Statistical analyses were done by using the SPSS program version 16 (IBM-USA). The one-way analysis of variance (ANOVA) test was used. When equal variance could be assumed, the LSD-t test was applied. Data were presented as mean \pm standard deviation (SD). $P<0.05$ was considered statistically significant.

\section{RESULTS}

\section{Constituents of AEC resulting from the GC-MS and HPLC analysis}

The major constituents of the cinnamon bark decoction are phenylpropanoids (12\%) and phenols (8\%). Other minor constituents are described in (Table 1).

\section{AEC prevented CDDP-induced Vero cell Toxicity}

Initially, the effect of AEC on the cell survival was determined using renal cell line, Vero, as a model. The cells were incubated with increasing concentrations $(0$, $10,20,40$, and $50 \mu \mathrm{g} / \mathrm{mL}$ ) of AEC for $24 \mathrm{~h}$, before being harvested and assayed for cell viability by WST-1 test. As shown in Fig. 1A, AEC marginally altered the basal level of the Vero cell viability, even at the level of $50 \mu \mathrm{g}$, AEC did not show toxicity. Thus, we concluded from these experiments that AEC is not toxic to normal Vero cells at the level as high as $50 \mu \mathrm{g} / \mathrm{mL}$.

To find whether cinnamon ameliorates or exacerbates the side effects of therapy in normal cells, we determined the effects of AEC on CDDP-induced kidney cell viability. The results summarized in Fig. 1B show incubation of Vero cells with increasing concentrations of CDDP for $24 \mathrm{~h}$. Generally, CDDP consistently exhibited a dosedependent anti-proliferative effect on the cell viability. The $\mathrm{IC}_{50}$ value (the concentration of test compound that inhibits $50 \%$ of the cell growth) for 10, 15, 20 and $25 \mu \mathrm{g} /$ $\mathrm{mL}$ of CDDP was around $15 \mu \mathrm{g} / \mathrm{mL}$ observed after $24 \mathrm{~h}$ of treatment, respectively.

Next, we incubated Vero cells with increasing doses of CDDP along with $25 \mu \mathrm{g} / \mathrm{mL}$ of AEC for $24 \mathrm{~h}$. As seen in

Table 1. The percent composition of different chemical categories in the cinnamon bark decoction.

\begin{tabular}{lc}
\hline Chemical category & \% composition \\
\hline Phenylpropanoids & 12 \\
Phenols & 8.0 \\
B-Cadinene & 2.6 \\
$\alpha$-Muurolen & 0.8 \\
$\alpha$-cadinene & 0.2 \\
Hydrocarbons & 0.3 \\
\hline
\end{tabular}




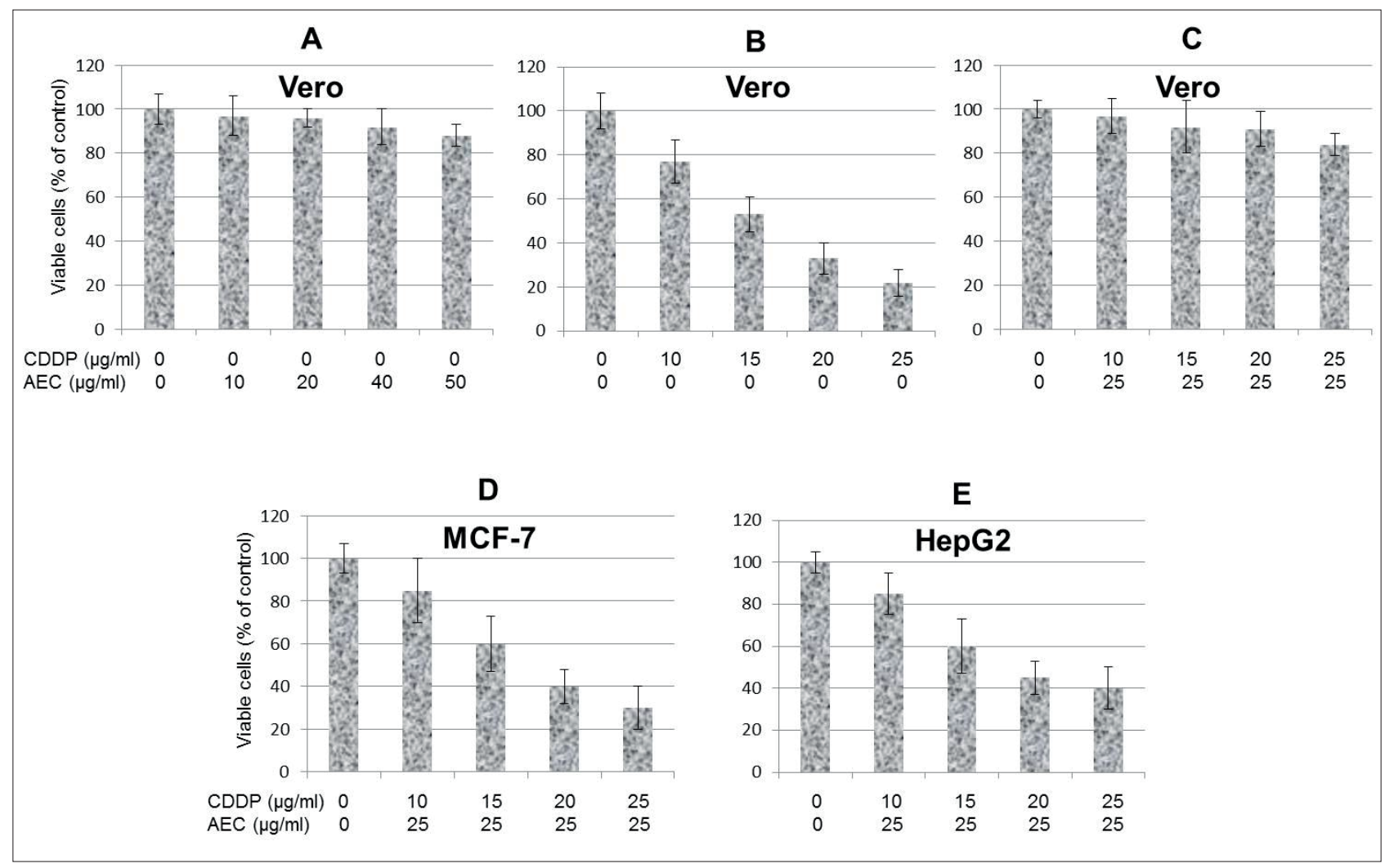

Fig. 1. AEC Prevented CDDP-Induced Vero Cell Toxicity without Compromising the Anticancer Efficiency of Cisplatin. Viability of Vero cells (A, B and C) were assayed after treatment with either AEC, CDDP or combination of both AEC and CDDP for 24 h. Viability of MCF-7 breast (D) and HepG2 hepatocellular (E) cancer cells were assayed after treatment with combination of $\mathrm{AEC}$ and CDDP for $24 \mathrm{~h}$. The experiments were repeated three times in triplicates, and values are reported as the mean \pm SD.

Fig. 1C, pre-incubation of the cells with AEC significantly increased the percentage of cell viability. The cytoprotective effects of AEC during CDDP treatment were dosedependent, and significant cytoprotection was shown at $10-50 \mu \mathrm{g} / \mathrm{mL}$ (Fig. 1C). These finding suggests that the treatment of Vero cells by AEC may play a key role in the protection against CDDP mediated toxicity.

To find whether AEC compromise cytotoxicity of CDDP induced cytotoxicity of cancer cells, the breast cancer cells (MCF-7) and hepatocellular carcinoma cells (HepG2) were treated with CDDP and AEC for $24 \mathrm{~h}$ and cell viabilities were assayed. As shown in Fig. 1, the AEC did not alter cytotoxicity of CDDP in case of MCF-7 (Fig. 1D) or HepG2 (Fig. 1E) cells, since the $\mathrm{IC}_{50}$ values of CDDP in the contexts of both cell lines were comparable to that for Vero cells (i.e. $15 \mu \mathrm{g} / \mathrm{mL}$ ). Thus, AEC has the potential to prevent CDDP Vero cell toxicity without compromising the anticancer efficacy of CDDP.

\section{AEC prevented CDDP-induced apoptotic cell death}

To determine whether AEC inhibits the CDDPdependent apoptotic cell death in Vero cells and whether AEC-abrogating CDDP-apoptosis potentiality accounts for its cyto-protective ability, cells were treated with increasing concentrations of AEC and CDDP for $24 \mathrm{~h}$ and the frequency of apoptotic cell death was assessed by phase microscopy. The findings in Fig. 2 display that control Vero cells appeared as a confluent layer of typical spindle-shaped cells adjacent to each other forming a confluent monolayer. They have well-defined outline and abundant cytoplasm, while the central nucleus revealed multiple recognizable nucleoli (Fig. 2A). On the other hand, cells treated with CDDP exhibited, in a dose-dependent manner, characteristic features of apoptosis. Some cells showed a rim of perinuclear halo and multiple cytoplasmic vacuoles of varying sizes and the nuclei appeared condensed and hyperchromatic. At higher concentrations, more cardinal signs of apoptosis were quite evident. These include cellular shrinking, membrane blebbing and condensation of the peripheral heterochromatin and cleavage of the nucleus and cytoplasm into multiple membraneenclosed apoptotic bodies. Importantly, CDDP-induced apoptosis was suppressed by AEC. To confirm the results, we analyzed apoptosis utilizing fluorescent nuclear stains. Staining of apoptotic cells with fluorescent dyes such as Ao and EtBr is considered the correct method for evaluating the changed nuclear morphology. In this assay, only $\mathrm{AO}$, but not $\mathrm{EtBr}$, can cross the plasma membrane of vital cells and stain the nuclei green. When the plasma membrane is disrupted at late apoptotic phase or due to necrosis, EtBr can also enter the cell and stain the nuclei red. Based on this, live cells will show a normal green nucleus. Early apoptotic cells show bright green nucleus owing to condensation of chromatin, while necrotic cells show 


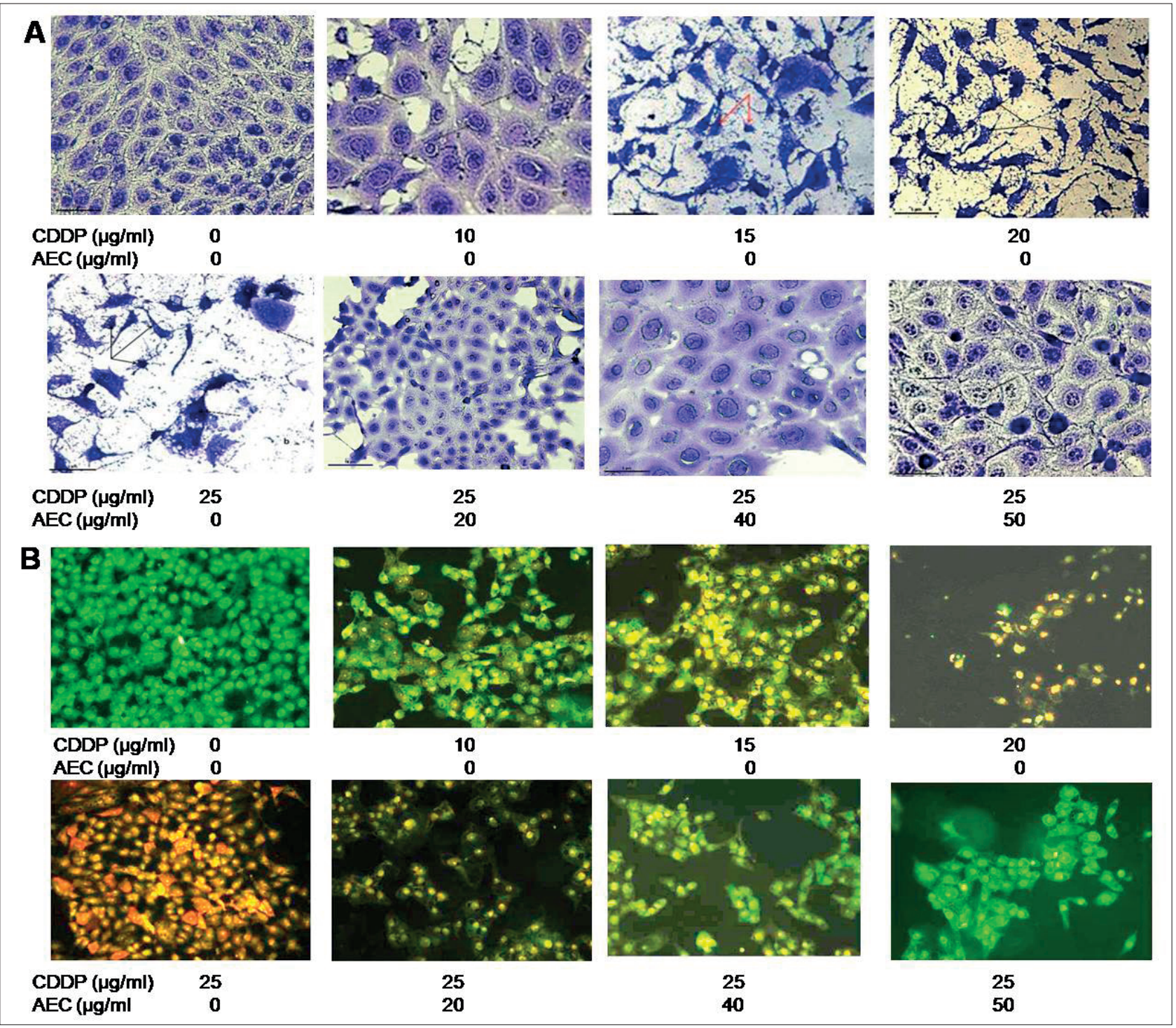

Fig. 2. AEC Prevented CDDP-Induced Apoptotic Cell Death. (A) Light microscope images displaying cells fixed in formalinbuffered phosphate and stained with Coomassie blue; notice gradual alteration of cell shapes as a result of increasing doses of CDDP. These include cellular, irregularity of cell shape and blebbing of cell membrane, condensation of chromatin, formation of apoptotic bodies (red \& black arrows) and late apoptosis/secondary necrosis; magnification 20x. (B) Cells were stained with vital stain ( $\mathrm{Ao} / \mathrm{EtBr}$ ) for $15 \mathrm{~min}$ at $37^{\circ} \mathrm{C}$ and visualized by florescent microscope. Note, treatment with induced apoptotic signs in Vero cells and including AEC with CDDP abrogated apoptotic signs induced by CDDP; magnification 10x.

intact normal red nucleus. In untreated cells, the nuclei fluoresced a faint green homogenous color, while in the treated cells the green emission was much brighter than in the unaffected ones owing to chromatin condensation. Our results depicted in Fig. 2B indicate that the nuclei of untreated control cells were found to be intact, round in shape and were stained green. In contrast, cells treated with 10 and $15 \mu \mathrm{g} / \mathrm{mL}$ CDDP had some cells stained green and some orange nuclei indicating an emergence of early apoptotic signs in the treated cells. At the highest doses of CDDP (20 and $25 \mu \mathrm{g} / \mathrm{mL}$ ), an increase in the number of cells with red-colored nuclei (necrotic/late apoptotic) was predominant. Thus, the morphological analysis of $\mathrm{AO} / \mathrm{EtBr}$ stained Vero cells indicated significant morphological changes.

\section{AEC prevented CDDP-induced activation of Bax and Caspase- 3 and release of cytochrome $c$}

To determine the effect of AEC on CDDP-induced Bax activation and cytochrome c release, Vero cells were treated with CDDP and AEC for $24 \mathrm{~h}$, then expression levels of both proteins in the cytosolic and mitochondrial compartments were assayed. As shown in Fig. 3A, in control cells, the majority of Bax was detected in the cytosolic fraction (lane 1), and, after CDDP treatment, a significant amount of Bax moved to the mitochondrial fraction (lane 2). Importantly, AEC effectively suppressed CDDP-induced Bax accumulation in mitochondria (lane 3). Meanwhile, CDDP treatment significantly induced the release of mitochondrial cytochrome $\mathrm{c}$ into cytosolic compartment, which was accompanied by the reduction of the cytochrome $\mathrm{c}$ in mitochondrial fraction 


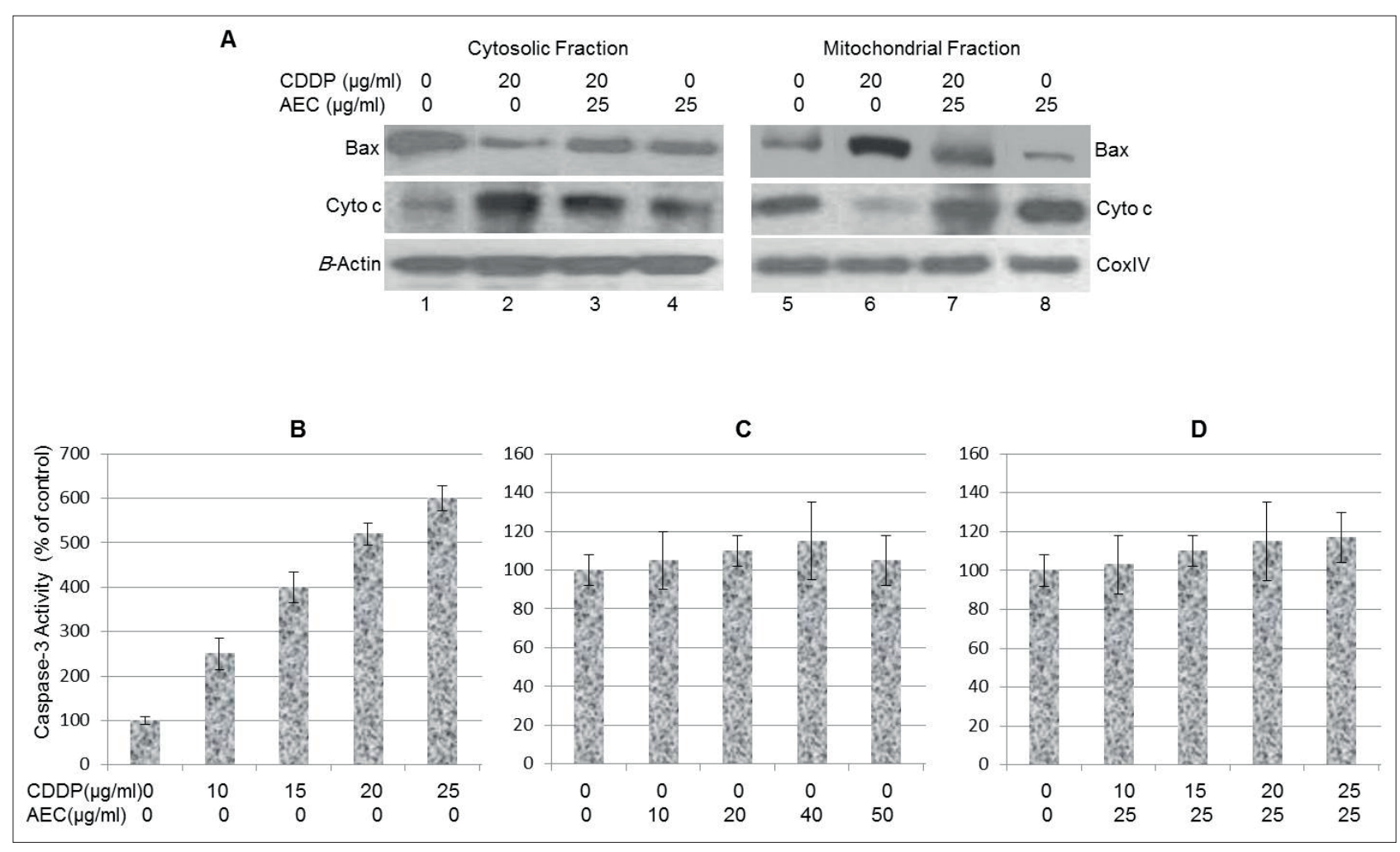

Fig. 3. AEC prevented CDDP-induced activation of Bax and Caspase- 3 and release of cytochrome c. (A) Left panels: immunoblot analysis of Bax translocation to mitochondria. Right Panels: immunoblot analysis of cytochrome c release. The immunoblots show in control cells, the majority of Bax was detected in the cytosolic fraction (lane 1), and, after CDDP treatment, a significant amount of Bax moved to the mitochondrial fraction (lane 6). Importantly, AEC effectively decreased potentiality of CDDPinduced Bax accumulation in mitochondria (lane 7). Meanwhile, cytochrom c (cyto c) is accumulated in mitochondrial fraction (lane 5) and CDDP treatment significantly induced release of mitochondrial cytochrome c into cytosolic compartment (lane 2), which was accompanied by reduction of the cytochrome $\mathrm{c}$ in mitochondrial fraction (lane 6). AEC alone did not induce Bax accumulation or cytochrome c release (lanes 4 and 8). (B-D) Histograms showing CDDP remarkably induced caspase-3 activity, in a dose-dependent manner (B); AEC insignificantly affected basal level of caspase-3 activity (C), and AEC abrogated potentiality of CDDP-induced caspase-3 activation (D).

(lane 4). AEC alone did not induce Bax accumulation or cytochrome c release (lanes 4 and 8 ), an observation that was consistent with the low toxicity of AEC in Vero cells. Together, these results suggest that AEC may protect against CDDP-induced apoptosis by inhibiting Bax activation and subsequent cytochrome c release.

Next, we investigated whether AEC could inhibit CDDP-induced caspase- 3 activation. We noticed that CDDP induced an increase in the activation of caspase-3, in a dose-dependent manner (Fig. 3B). Remarkably, AEC alone did not have any effect on the activation of caspase-3 (Fig. 3C); in addition, it significantly suppressed CDDP-induced caspase- 3 activation in a dose-dependent manner (Fig. 3D).

\section{AEC prevented CDDP-induced ROS generation and DNA fragmentation, and mediated up-regulation of HO-1 expression}

To determine whether AEC treatment might abrogate CDDP-induced ROS generation, Vero cells were treated with CDDP, AEC or both agents for $24 \mathrm{~h}$, then incubated with a fluorescent dye, H2DCFDA, for $60 \mathrm{~min}$ and then fluorescence intensity was recorded. As seen in Figure 4A, CDDP treatment promoted basal level of ROS in
Vero cells. On the other hand, when AEC was added into the growth medium, along with CDDP, ROS generation decreased to the basal level. In addition, AEC treatment alone insignificantly altered the basal level of ROS.

Next, DNA fragmentation was quantitated after single and double treatment with AEC and CDDP. The percentage of DNA fragmentation dose-dependently increased after incubation of the cells with CDDP (Fig. 4B). However, when cells were treated with CDDP along with AEC, we could not observe DNA fragmentation (Fig. 4B). Therefore, we concluded from these experiments that AEC has a potentiality to suppress CDDP-induced DNA fragmentation leading to apoptosis.

Finally, we investigated whether the protective effect of AEC is mediated by the induction of HO-1 expression in Vero cells. The cells were treated with CDDP, AEC or both agents, and the expression level of HO-1 was assessed by reverse transcription-PCR. We observed that the HO-1 mRNA was insignificantly down-regulated in the CDDP-treated cells compared with the control (Fig. 4C). However, when the cells were treated with AEC, along with CDDP, HO-1 mRNA expression level was remarkably up-regulated. This finding suggests that the induction of HO-1 by AEC treatment may play a key role 
in the protection against CDDP-mediated Vero cell toxicity. Collectively, these data suggest that CDDP induced oxidative stress in Vero cells, whereas such stress was significantly blocked by the AEC treatment.

\section{DISCUSSION}

A major issue in cancer therapy is the potential side effects and toxicity to normal tissues. For example, CDDP is a highly prescribed drug for cancer; nonetheless, its chemotherapeutic efficacy is severely limited by its nephrotoxicity ${ }^{2}$. Therefore, there is an urgent need to identify agents with potentiality to suppress the cytotoxic effect of CDDP. Several plant-derived phytochemicals have been found to prevent CDDP-induced nephropathy ${ }^{28,29}$. In the current study, we demonstrated that the aqueous extract of the herb, $C$. cassia (AEC), can protect normal cells against CDDP-induced cytotoxicity in Vero cells. Significantly, AEC had no noticeable effect on the anticancer efficiency of CDDP against MCF-7 and HepG2 cells. Thus, AEC has the potential to suppress CDDP toxicity in normal Vero cells without compromising the anticancer efficacy of cisplatin. The data here show that cells treated with CDDP exhibited morphological hallmarks of cells committing apoptotic cell death; these include cellular shrinkage and detachment, condensed and hyperchromatic nuclei surrounded by a perinuclear halo and formation of apoptotic bodies. These observations indicate that under our experimental conditions (applied doses and treatment intervals), the reduction of cell number after CDDP treatment is due to apoptotic cell death.

A major finding of this study is that AEC protects against CDDP-induced apoptotic cascades in Vero cells. It is well-known that CDDP exerts its apoptotic activity through activation of the intrinsic (mitochondrial) pathway (4). A mainstay event in this pathway is the permeabilization of the outer mitochondrial membrane allowing several pro-apoptotic factors, such as cytochrome c, to be released into the cytosol ${ }^{5}$. The release of cytochrome c, however, is tightly regulated by members of a Bcl-2 protein family ${ }^{30}$; the Bcl- 2 protein prevents, whilst the Bax protein boosts release of the cytochrome. In healthy cells, Bax exists in an inactive form mainly in the cytosol; upon activation by apoptotic signals, it migrates to the mitochondria and boosts release of the cytochrome $\mathrm{c}$ into cytosol ${ }^{30}$. The released cytochrome $\mathrm{c}$ mediates, along with other cytosolic proteins, activation cascade of caspases including caspase-9, which subsequently activates further downstream caspases including caspase-3. Caspase- 3 in particular is one of the key executioners of apoptosis and is either partially or wholly responsible for the proteolytic cleavage of many proteins and DNA fragmentation, ultimately leading to cell death ${ }^{5}$. Accumulative studies demonstrate that CDDP triggers all these (abovementioned) activities to induce apoptosis ${ }^{2}$. Therefore, in the present study, we monitored the translocation of Bax, cytochrome c release, activation of caspase- 3 and cleavage of DNA to better understand the molecular mechanisms involved. Consistent with earlier studies, our results showed that CDDP treatment resulted in translocation of Bax, release of cytochrome c, progressive activation of caspase- 3 and

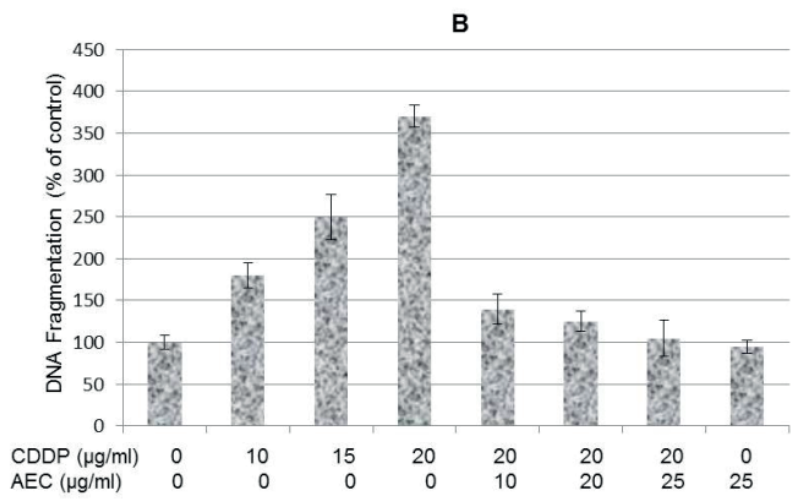

A

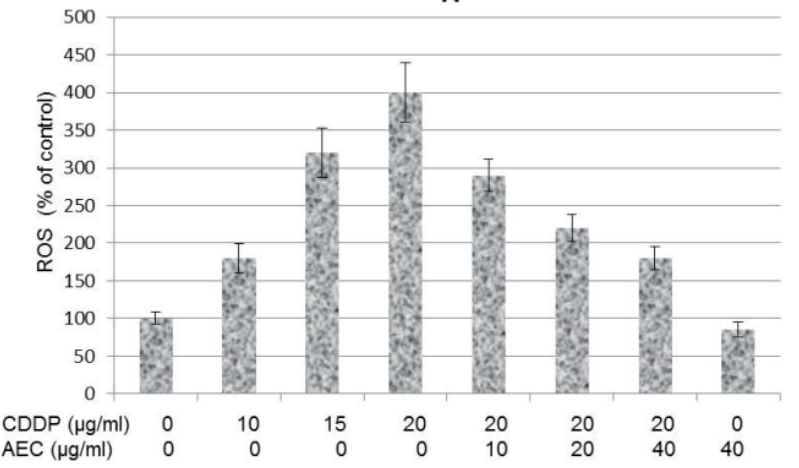

C

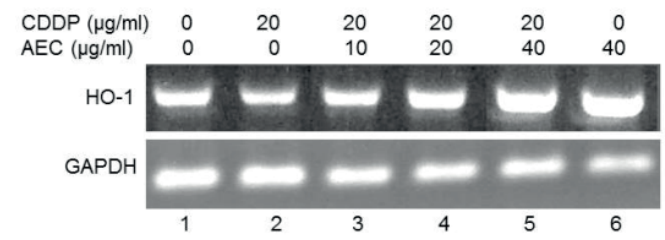

Fig. 4. AEC prevented CDDP-induced ROS generation and DNA fragmentation, and mediated up-regulation of HO-1 expression. (A) The results show that CDDP remarkably induced ROS production, in a dose-dependent manner, AEC abrogated potentiality of CDDP-induced ROS generation. (B) Percentage of DNA fragmentation as determined by the diphenylamine assay. The experiments were repeated three times in triplicates, and values are reported as the mean \pm SD. (C) Vero cells were treated with CDDP and/or AEC for $24 \mathrm{~h}$. The results show that CDDP reduced level of HO-1 compared to the control, AEC promoted level of HO-1 mRNA, in dose-dependent manner. The experiments were repeated three times in triplicates, and values are reported as the mean $\pm \mathrm{SD}$.

fragmentation of DNA. Significantly, we found that all these activities were obviated, when cells were treated with CDDP along with AEC. Thus, this study has uncovered a new pharmacological function of AEC, blocking CDDP-induced apoptotic cascades. This finding is important because it suggests that AEC may enhance therapeutic efficacy during combinational therapies with CDDP by alleviating the side effects of CDDP in normal tissues.

Substantial evidence indicates that the production of ROS by CDDP in the kidney is crucial to the progression of nephrotoxicity ${ }^{31}$. The ROS are seen as early signs partially responsible for the activation of various signaling 
pathways that culminate in kidney failure, renal injury, and cell death in the event of CDDP-induced nephrotoxicity $^{2}$. The data here show that AEC treatment prevents the generation of ROS by CDDP, which could be another mechanism underlying the protective potentiality of AEC.

A large number of studies report that oxidative stress stimulates kidney cells to produce a cytoprotective response. This is best illustrated by HO-1, an enzyme with potent antioxidant activity, and upregulation of HO-1 expression is recognized as a key event in maintaining cellular antioxidant capacity. HO-1-deficient rats were significantly more sensitive to CDDP-induced kidney damage compared to wild controls. HO-1 overexpression significantly reduced in vitro CDDP-induced apoptosis, albeit the molecular basis for the cryoprotective effects of HO-1 is not entirely clear ${ }^{32}$. Consistent with this, the genetic depletion or chemical inhibition of HO-1 significantly worsens kidney function and tubular injury in response to CDDP treatment, further supporting a protective role for HO-1 expression in CDDP-induced renal injury ${ }^{33}$. In addition, various antioxidants have the ability to effectively protect against CDDP-induced nephrotoxicity ${ }^{34}$. Likewise, cinnamon has been demonstrated to possess antioxidant activities ${ }^{12}$, and thus, be potentially protective against CDDP-mediated apoptosis. In this study, we found that AEC treatments promoted the expression of HO-1 mRNA, which was associated with the reduction of CDDP-mediated cell death. Therefore, up-regulation of HO-1 by AEC suggest that AEC treatment might trigger events leading to attenuation of apoptotic cascades, which may contribute, at least in part, to the reduction of CDDP-induced cytotoxicity in Vero cells. Therefore, the protective effect of AEC has a potential in combinational therapy.

\section{CONCLUSION}

This study in Vero cells reveals several molecular mechanisms that could explain why AEC prevents CDDPinduced Vero cell death. AEC prevented apoptotic cell death at multiple levels: (1) AEC prevented Bax activation and translocation from the cytosolic to the mitochondrial compartment; (2) AEC prevented the release of cytochrome $\mathrm{c}$ from the mitochondria to the cytosolic compartment; (3) AEC ameliorated CDDP-induced caspase-3 activation; (4) prevented CDDP-induced ROS generation, and (5) up-regulated HO-1 mRNA expression. Altogether, these intracellular events resulted in attenuation of apoptosis. The present study suggests that the inhibition of apoptosis and activation of HO-1 by the regular intake of the natural phytochemical "cinnamon" may be one of the most practical and feasible ways to reduce the risk of CDDP-induced toxicity.

\section{ABBREVIATIONS}

$\mathrm{AO}$, Acridine orange; AEC, Aqueous extract of cinnamon; CDDP, Cisplatin; EtBr, Ethidium bromide;

HO-1, Heme oxygenase; ROS, Reactive oxygen species.

Acknowledgment: This work was funded by the Deanship of Scientific Research (DSR), King Abdulaziz University, Jeddah, under grant No. 140-005-D1433. The authors are grateful to DSR for their technical and financial support. Special appreciation goes to Miss Hanadi Fouad Qashqari, lecturer and assistant researcher at KFMRC for her tremendous help at the Tissue Culture Unit at KFMRC-KAU.

Author contributions: WR: owner of the idea, prepared the aqueous extract, has done the photographing using light microscope, performed the statistical analysis, and shared in the writing and revision processes. AE: carried out the molecular studies, photographing using fluorescent microscope, discussing the results, and shared in the writing and revision processes. Both authors: final approval.

Conflict of interest statement: The authors state that there are no conflicts of interest regarding the publication of this article.

\section{REFERENCE}

1. Boulikas T, Vougiouka M. Cisplatin and platinum drugs at the molecular level (Review). Oncol Rep 2003;10:1663-82.

2. Ozkok A, Edelstein CL. Pathophysiology of Cisplatin-Induced Acute Kidney Injury. Biomed Res Int. 2014;2014:967826. doi: $10.1155 / 2014 / 967826$

3. Launay-Vacher V, Rey JB, Isnard-Bagnis C, Deray G, Daouphars M. European Society of Clinical Pharmacy Special Interest Group on Cancer Car. Prevention of cisplatin nephrotoxicity: state of the art and recommendations from the European Society of Clinical Pharmacy Special Interest Group on Cancer Care. Cancer Chemother Pharmacol 2008;61:903-9.

4. Peres LA, da Cunha AD Jr. Acute Nephrotoxicity of Cisplatin: Molecular mechanisms. J Bras Nefrol 2013;35(4):332-40.

5. Wong RS. Apoptosis in cancer: from pathogenesis to treatment. J Exp Clin Cancer 2011; Res 30:87.

6. Sarastea A, Pulkki K. Morphologic and biochemical hallmarks of apoptosis. Cardiovascular Research 2000;45:528-37.

7. Yin SY, Wei WC, Jian FY, Yang NS. Therapeutic applications of herbal medicines for cancer patients. Evid Based Complement Alternat Med 2013;2013:302-426.

8. Jakhetia V, Pate R, Khatri P, Pahuja N, Garg S, Pandey A, Sonu Sharma S. Cinnamon: A Pharmacological Review. Journal of Advanced Scientific Research 2010;1(2):19-23.

9. Zou YH, Liu XM. Effect of astragalus injection combined with chemotherapy on quality of life in patients with advanced non-small cell lung cancer. Zhongguo Zhong Xi Yi Jie He Za Zhi 2003;23:733-5.

10. Han MQ, Su JM, Huang HY, Long WH, Qin LP, Qin P, Liu W, Sheng ZY. Prognostic analysis of advanced non small cell lung cancer treated by sequential chemo-radiation therapy combined with traditional Chinese medicine: a report of 54 cases. Zhong Xi Yi Jie He Xue Bao 2003;1:195-8.

11. Chaudhry NMA, Tariq P. Anti-microbial activity of cinnamomum cassia against diverse microbial flora with its nutritional and medicinal impacts. Pak J Bot 2006;38(1):169-74.

12. Sharma V, Rao LJ. An Overview on Chemical Composition, Bioactivity and Processing of Leaves of Cinnamomum tamala. Critical Reviews in Food Science and Nutrition 2014;54:433-48.

13. Vangalapati M, Sree Satya N, Surya Prakash DV, Avanigadda S. 
Review on Pharmacological Activities and Clinical effects of Cinnamon Species. Research Journal of Pharmaceutical, Biological and Chemical Sciences 2012;3(1):653-63.

14. Shobana S, Akhilender Naidu K. Antioxidant activity of selected Indian spices. Prostaglandins Leukotrienes and Essential Fatty Acids 2000;62(2):107-10.

15. Okawa M, Kinjo J, Nohara T, Ono M. DPPH (1,1-diphenyl-2-Picrylhydrazyl) radical scavenging activity of flavonoids obtained from some medicinal plants. Biological and Pharmaceutical Bulletin 2001;24(10):1202-5.

16. Shan B, Cai YZ, Sun M, Corke H. Antioxidant capacity of 26 spice extracts and characterization of their phenolic constituents. J Agric Food Chem 2005;53(20):7749-59.

17. Lu J, Zhang K, Nam S, Anderson RA, Jove R, Wen W. Novel angiogenesis inhibitory activity in cinnamon extract blocks VEGFR2 kinase and downstream signaling. Carcinogenesis 2010;31(3):481-8.

18. Kwon B-M, Lee S-H, Cho Y-K, et al. Synthesis and biological activity of cinnamaldehydes as angiogenesis inhibitors. Bioorganic and Medicinal Chemistry Letters 1997;7(19):2473-6.

19. Jayaprakasha GK, Rao LJ. Chemistry, biogenesis, and biological activities of Cinnamomum zeylanicum. Crit Rev Food Sci Nutr 2011;51(6):547-62.

20. Mohamed AM, Metwally NS. Antiaflatoxigenic Activities of Some Plant Aqueous Extracts Against Aflatoxin-B1 Induced Renal and Cardiac Damage. Journal of pharmacology and toxicology 2009;4(1):1-16.

21. Li J, Dong J, Qiu JZ, Wang JF, Luo MJ, Li HE, Leng BF, Ren WZ, Deng XM. Peppermint oil decreases the production of virulenceassociated exoproteins by Staphylococcus aureus. Molecules 2011:16(2):1642-54.

22. Ding $Y$, Wu EQ, Liang $C$, Chen J, Tran MN. Hong CH, Jang Y, Park KL, Bae K, Kim YH, Kang JS. Discrimination of cinnamon bark and cinnamon twig samples sourced from various countries using HPLC-based fingerprint analysis Food Chem 2011;127(2):755-60.

23. Santos AR, Dolder H, Wada ML. Dexamethasone and fetal calf serum effects in differentiation of Vero cells cultured on type I collagen gel. J Submicrosc Cytol Pathol 2003;35(1):35-42.

24. Estacia P, Santos AR Jr, Moreira PL, Genari SC. The Cytotoxicity In Vero Cells Of A Perfluorocarbon Used In Vitreoretinal Surgery. Braz J Morphol Sci 2002;19(2):41-7.

25. Abid-Essefi S, Bouaziz C, Golli-Bennour EE, Ouanes Z, Bacha H. Comparative Study of Toxic Effects of Zearalenone and Its Two Major Metabolites $\alpha$-Zearalenol and $\beta$-Zearalenol on Cultured Human Caco-2 Cells. J Biochem Mol Toxicol 2009;23(4):233-43.

26. Elkady Al. Crude alkaloid extract of Rhazya stricta inhibits cell growth and sensitizes human lung cancer cells to cisplatin through induction of apoptosis. Genetics and Molecular Biology 2013;36(1):12-21.

27. Jung SH, Kim HJ, Oh GS, Shen A, Lee S, Choe SK, Park R, So HS. Capsaicin Ameliorates Cisplatin-Induced Renal Injury through Induction of Heme Oxygenase-1. Mol Cells 2014;37(3):234-40.

28. Ju SM, Kang JG, Bae JS, Pae HO, Lyu YS, Jeon BH. Flavonoid Apigenin Ameliorates Cisplatin-Induced Nephrotoxicity through Reduction of p53 Activation and Promotion of PI3K/Akt Pathway in Human Renal Proximal Tubular Epithelial Cells. The Evid Based Complement Alternat Med 2015; 2015;2015:186436. doi: 10.1155/2015/186436

29. Kim TW, Kim YJ, Park SR, Seo CS, Ha H, Shin HK, Jung JY. Chrysanthemum indicum attenuates cisplatin-induced nephrotoxicity both in vivo and in vitro Nat Prod Commun 2015;10(3):397-402.

30. Reed JC. Mechanisms of Apoptosis. Am J Pathol 2000;157(5):141530.

31. Sahu BD, Kuncha M, Sindhura GJ, Sistla R. Hesperidin attenuates cisplatin-induced acute renal injury by decreasing oxidative stress, inflammation and DNA damage. Phytomedicine 2013;20:453-60.

32. Tayem Y, Johnson TR, Mann BE, Green CJ, Motterlini R. Protection against cisplatin-induced nephrotoxicity by a carbon monoxidereleasing molecule. Am J Physiol Renal Physiol 2006;290:789-94.

33. Agarwal A, Balla J, Alam J, Croatt AJ , Nath KA. Induction of heme oxygenase in toxic renal injury: a protective role in cisplatin nephrotoxicity in the rat. Kidney International 1995:48(4):1298-1307.

34. Pabla N, Dong Z. CDDP nephrotoxicity: mechanisms and renoprotective strategies. Kidney Int 2008;73(9):994-1007. 